\title{
ESTUDIO EXPLORATORIO SOBRE GESTIÓN DE CONVIVENCIA EN CENTROS CON BUENAS PRÁCTICAS
}

\section{Sara Conde Vélez y José Antonio Ávila Fernández}

\section{RESUMEN}

El objetivo de este estudio es la descripción de factores que inciden en la mejora de la convivencia escolar realizando un estudio exploratorio sobre centros de convivencia escolar con buenas prácticas en Andalucía a través del Análisis de Correspondencia Múltiple (ACM en adelante). La muestra la componen miembros de la comisión de convivencia de 46 centros educativos pertenecientes a la red «Escuela: Espacio de Paz». Para la recogida de datos se elaboran cinco escalas ad hoc. Una de las principales conclusiones a la que se llega con este estudio es que la reducción de problemas de convivencia en los centros educativos aparece asociados con altos valores en la gestión de medidas preventivas, sobre todo a nivel primario o secundario.

Palabras clave: Convivencia escolar, gestión de la convivencia escolar, problemas de convivencia, mediación de conflicto.

\section{TITLE: AN EXPLORATORY STUDY ON THE MANAGEMENT OF COMMUNITY LIFE IN EDUCATIONAL CENTERS WITH GOOD PRACTICE}

\section{ABSTRACT}

The objectives of this study are summarized in the description of factors that appear to influence the improvement of school life by performing an exploratory study on school life in centers with good practice in Andalusia through Multiple Correspondence Analysis (MCA onwards). The sample is made up of members of the coexistence committee of 46 schools belonging to the 'School: Space of Peace' network. For data collection five scales are developed ad hoc. One of the main conclusions that can be reached with this study is that reducing problems of coexistence in schools, appears to be associated with high values in the management of preventive measures, especially at the primary or secondary level.

Keywords: Community life at school, management of community life at school, problems of coexistence, conflict mediation.

Correspondencia con los autores: Sara Conde Vélez. < sara.conde@dedu.uhu.es>. José Antonio Ávila Fernández. $<$ jaavila@dedu.uhu.es>.Departamento de Educación, Universidad de Huelva, . Original recibido: 27-I2-13. Original aceptado: 17-03-14 


\section{Introducción y marco teórico}

Son muchos los problemas de convivencia existentes dentro de las escuelas de España, fenómeno también extensible a diferentes países de la Unión Europea (Cangas, Gázquez, Pérez, Padilla y Miras, 2007). Concretamente en España, son de destacar los resultados del estudio estatal sobre convivencia escolar en la educación secundaria obligatoria, el informe anual sobre el estado de la convivencia en los centros educativos de Andalucía sostenidos con fondos públicos durante el curso 2010-20II, así como diversos trabajos empíricos. En muchos estudios se subraya como principales problemas de convivencia (Andrés y Barrios, 2009; Ávila, 2013; Conde, 2012; DíazAguado, Martínez y Martín, 20I0; Fernández-Enguita, 2007; Pérez de Guzmán y Amador, 20I I; Sheerens y Bosker, 1997): conductas disruptivas, consideradas como aquellas acciones que dificultan el normal desarrollo de las clases; indisciplina en las relaciones entre el profesorado y el alumnado; problemas relacionales entre el alumnado, como vandalismo, daños materiales, extorsiones, agresiones, violencia física, etc.; falta de implicación familiar; desmotivación y desinterés académico, tanto por parte del alumnado como del profesorado; absentismo; el hecho de ser percibido diferente, entre otros.

De acuerdo con Rodríguez (2007), las respuestas ofrecidas por las Administraciones y por los centros educativos están orientadas a la creación de programas de prevención de la violencia desde la concepción de la escuela como una organización que convive con el conflicto.

A continuación se plantean cuatro factores que parecen incidir en la mejora de la convivencia escolar, en los que se centra este estudio. Estos factores se corresponden con medidas a nivel de gestión de centro y principios de actuación que la literatura científica y las normativas en materia de educación han puesto de relieve por su influencia sobre la reducción de los problemas de convivencia.

\section{I. Liderazgo}

Leithwood (2009) define el liderazgo escolar como la labor de movilizar e influenciar a otros para articular y lograr intenciones y metas compartidas en la escuela. Más concretamente, Torrego (2010) afirma que este liderazgo debe entenderse desde una perspectiva crítica que acentúe el carácter crítico, comprometido, transformador y ético del mismo que se traduzca en la formación de una «Comunidad Profesional». 
En la literatura, se identifica el liderazgo como uno de los factores críticos de éxito para mantener la mejora continua en cualquier organización (Taffinder, 1995). Evidencias empíricas, como las aportadas en los estudios de Eskildsen y Dahlgaard (2000) y Flynn, Schroeder y Sakakibara (1994), muestran una correlación positiva entre el estilo de liderazgo, caracterizado por la participación de la comunidad educativa, $y$ otras variables que favorecen un clima de convivencia positivo.

\section{I.2. Formación de la comunidad}

En general, puede afirmarse como una línea de actuación preferente la implantación de un modelo pedagógico entendido desde la atención a la diversidad y el aprendizaje cooperativo, desde la profundización en la educación en valores, con la presencia de técnicas de mediación y resolución pacífica de conflictos, en el que las familias se impliquen en las tareas educativas, orientado desde la autonomía pedagógica y comprometido con la formación permanente del profesorado (García y López, 20I I).

Un modelo educativo de esta naturaleza pasa por la formación de la comunidad escolar: discentes, familiares y docentes. Ortega, Romera y Del Rey (2005) ofrecen una serie de claves para una respuesta preventiva a la violencia escolar, entre las que destaca la formación del profesorado. Este elemento se convierte en uno de los pilares fundamentales para dar respuesta a la violencia escolar y promover la convivencia.

\section{I.3. Educar en el conflicto}

Se muestra cada vez más evidente la necesidad de aprovechar situaciones educativas que permitan aprender a vivir y disfrutar de una convivencia no exenta de conflictos y problemas. Igualmente, los conflictos entendidos como fenómenos sociales son oportunidades (Bodine y Crawford, 1998), que pedagógicamente aprovechadas permiten el desarrollo de competencias para ejercer una ciudadanía activa, crítica y en permanente crecimiento.

Ya se puso en evidencia en el meta-análisis realizado por Johnson y Johnson (1996) de investigaciones en resolución de conflictos y los programas de mediación entre pares, así como en estudios más recientes como los de Hendry (2010) y Maxwell (2007), que la resolución de conflictos y los programas de mediación 
entre compañeros parecen ser eficaces para enseñar a los estudiantes habilidades de negociación y procedimientos de mediación; incluso después de la capacitación, tienden a seguir utilizando estas estrategias de resolución de conflicto, que generalmente conducen a resultados constructivos, lo que llega a suponer una reducción del número de conflictos alumno-alumno relatados por los maestros y administradores, lo que en consecuencia logra reducir el número de expulsiones (Burton, 2012; Johnson y Johnson, 1996).

En este tipo de escenarios, las técnicas de resolución de conflictos como la mediación adquieren protagonismo frente al sistema sancionador resuelve los problemas exclusivamente en base a sanciones reguladas en los reglamentos de régimen interno de los centros (Peligero, 2010).

\subsection{Participación de toda la comunidad en la elaboración de las normas}

Las normas de convivencia han de introducir suficientes elementos que permitan prevenir los problemas de convivencia. Los reglamentos constituyen un reflejo del tipo de centro escolar. $Y$, en este sentido, se pueden identificar distintos tipos de normas (Casamayor, 2002): formalistas y rutinarias, autoritarias y rígidas, arbitrarias, y también creativas y dinámicas. Cuando la norma es creativa y dinámica, esta constituye un reflejo de escuela democrática, autónoma y participativa, que construye sus propias respuestas y contextualiza las pautas generales (Rodríguez, 2007 y Jordan, 2009). Desde este modelo la participación de toda la comunidad educativa en la elaboración de las normas, resulta un factor esencial como instrumento para la prevención de problemas de convivencia.

\section{I.5. Las «aulas de convivencia» como medida correctora}

Una de las medidas presentadas y regulada tanto en el Decreto de Convivencia 19/2007 como en la Orden de 20 de junio de 20I I, por la que se adoptan medidas para la promoción de la convivencia en los centros docentes sostenidos con fondos públicos y se regula el derecho de las familias a participar en el proceso educativo de sus hijos e hijas es la creación de aulas de convivencia para el tratamiento individualizado del alumnado que, como consecuencia de la imposición de una corrección o medida disciplinaria, se vea privado de su derecho a participar en el normal desarrollo de las actividades lectivas. El objetivo del Aula de Convivencia es convertirse en una alternativa a la expulsión del centro para aquellos alumnos y 
alumnas sancionados con esta medida. En estas aulas de convivencia se favorecerá un proceso de reflexión por parte de cada alumno o alumna que sea atendido en las mismas acerca de las circunstancias que han motivado su presencia en ellas. El plan de convivencia incluirá, en relación con el aula de convivencia, los siguientes aspectos:

a) Criterios y condiciones para la atención del alumnado en el aula de convivencia, profesorado que la atenderá y actuaciones que se desarrollarán en la misma, de acuerdo con los criterios pedagógicos que a tales efectos sean establecidos por el equipo técnico de coordinación pedagógica.

b) Programación de las actuaciones del departamento de orientación o del equipo de orientación educativa, según corresponda, encaminadas a favorecer un proceso de reflexión por parte de cada alumno o alumna que sea atendido en el aula de convivencia acerca de las circunstancias que han motivado su presencia en ella y el reconocimiento de su responsabilidad, para favorecer actitudes y conductas positivas para la convivencia.

c) Horario de funcionamiento del aula de convivencia.

d) Ubicación del aula, instalaciones y material didáctico con el que se cuenta para su funcionamiento.

Serrano, Tormo y Granados (20I I), tras los resultados obtenidos de un programa piloto sobre la implantación de las aulas de convivencia en diversos centros educativos de la Comunidad Valenciana, hacen notorio que el buen funcionamiento de las Aulas de Convivencia en estos centros depende de la utilidad de los materiales que en ella se usen, elaborados para lograr disminuir las conductas que alteran la convivencia escolar.

En definitiva, las aulas de convivencia resultan una novedosa opción para propiciar el desarrollo de actividades alternativas para aquellos alumnos o alumnas que, por presentar comportamientos disruptivos, se ven privados/as de continuar con el curso habitual de sus clases. Son una herramienta de trabajo para la formación del alumnado en convivencia, creando espacios de reflexión ante las actitudes disruptivas y fomentando actitudes empáticas (Serrano, Tormo, y Granados, 20II). 


\section{Objetivos}

Los objetivos de este estudio son:

I) Describir los factores que inciden en la mejora de la convivencia escolar.

2) Identificar tipos de centros en función de los factores medidos por cada una de las escalas.

3) Determinar la incidencia del tipo de centro en la mejora de la convivencia.

\section{Método}

\section{I. Participantes}

Para la selección de la muestra, se ha utilizado un muestreo intencional adoptando la antigüedad y experiencia como criterio objetivo. Concretamente la muestra se compone de 46 centros de educación secundaria de Andalucía integrados en la red «Escuela: Espacio de Paz» durante un periodo superior a 8 años, pertenecientes a las ocho provincias andaluzas (Tabla I).

\begin{tabular}{cc}
\multicolumn{2}{c}{ Muestra } \\
\hline Provincias & $\mathbf{N}^{\circ}$ de centros \\
\hline Almería & 4 \\
\hline Cádiz & 4 \\
\hline Córdoba & 3 \\
\hline Granada & 6 \\
\hline Huelva & 3 \\
\hline Jaén & 6 \\
\hline Málaga & 13 \\
\hline Sevilla & 7 \\
\hline TOTAL & 46 \\
\hline
\end{tabular}

Tabla I: Número de centros seleccionados por provincias 
En cuanto a su ubicación geográfica, los IES estudiados son diversos y heterogéneos, distribuidos tanto en zonas rurales como urbanas. No obstante la mayoría de los centros seleccionados se encuentran situados en zonas barrio y extrarradio de los diferentes municipios donde se ubican los IES, con un nivel económico cultural medio-bajo. En esta variable las zonas barrio y extrarradio suman el $81,1 \%$ de la muestra (37 centros).

Para la recogida de datos se optó por tomar como informantes a 46 profesores y 46 familiares que participan en las Comisiones de Convivencia de los centros de Educación Secundaria seleccionados.

\subsection{Procedimiento, instrumento y variables}

Para la recogida de datos se elaboran seis escalas ad hoc. A saber: «Liderazgo y Planificación Participada», «Elaboración Democrática de Normas», «Educación en el Conflicto», «Formación de Docentes y Familiares», «Aula de Convivencia» y «Efectos». Cada una de las mismas queda integrada por una serie de indicadores a valorar a través de una escala tipo Likert con valores comprendidos entre I y 4, siendo I nada, 2 algo, 3 bastante y 4 mucho, incluyendo también la opción no sabe/no contesta.

Las escalas utilizadas se muestran a continuación:

\section{Indicadores}

La Comisión de Convivencia elabora el método y los instrumentos para la recogida de información.

La Comisión de Convivencia realiza el diagnostico de la situación de la convivencia en el centro.

La Comisión de Convivencia, elabora una propuesta de contenidos del plan.

La Comisión de Convivencia recoge y discute información de todos y con todos los sectores de la comunidad educativa.

La Comisión de Convivencia acota los tiempos y personas responsables para el análisis de la convivencia.

La Comisión de Convivencia identifica los recursos humanos y materiales necesarios.

Existen procedimientos para explicar y difundir el plan de convivencia entre el personal

Existen procedimientos para explicar y difundir el plan de convivencia entre el alumnado.

Existen procedimientos para explicar y difundir el plan de convivencia entre las familias

Se explicita para cada una de las actividades: las personas responsables, los recursos, la metodología, contenido, espacio y temporización.

Tabla 2. Escala: Liderazgo y Planificación Participada 
164 Estudio exploratorio sobre gestión de convivencia en centros con buenas prácticas

\section{Indicadores}

Las normas son elaboradas contando con la participación de toda la comunidad educativa.

Se hace participar a toda la comunidad educativa en la identificación de los problemas de convivencia.

Se indaga sobre las causas de los problemas para establecer las normas.

Existen eficaces mecanismos para la difusión de las normas.

Las normas se revisan periódicamente.

Se establecen mecanismos para garantizar el conocimiento de las familias de las normas de convivencia.

Conocimiento que tienen las familias del plan de convivencia.

Tabla 3. Escala: Elaboración Democrática de Normas

\section{Indicadores}

Se elaboran materiales propios en relación con la convivencia

El material está organizado, clasificado y accesible para el profesorado

Se hacen actividades de acogida para informar sobre las normas de convivencia

Se estimula la participación de las familias en la vida del centro

Se favorece en el aula la participación activa de los estudiantes

Se realizan actividades que faciliten la reflexión del alumnado

Se hacen actividades que mejoren la actitud del alumno/a

Las actividades son adaptadas en función de las características del alumnado

El tutor analiza junto a la familia el progreso y cumplimiento de los acuerdos

Se acuerdan encuentros periódicos entre tutor y familiares para el seguimiento

Se identifica y define el conflicto

Se clarifica los intereses y sentimientos de las partes en el conflicto

Se generan alternativas para dar posibles soluciones

Se evalúa las alternativas y se toman decisiones

Se crean formas de cumplimiento de la solución

Queda claro el compromiso de cada persona

Cada parte expresa lo que aprendió y cómo puede aplicarlo

Se expresa cómo se han sentido y cómo se sienten con las soluciones

Se reconoce el esfuerzo y la contribución de las partes

Se hace un seguimiento para evaluar el cumplimiento de los acuerdos

Tabla 4. Escala: Educación en el Conflicto 


\section{Indicadores}

El equipo directivo establece estrategias e instrumentos para facilitar la participación en la elaboración del Plan

Se identifican las características del profesorado

Se elaboran recursos para la formación en mejora de convivencia escolar

Se impulsa la formación de familiares en mejora de la convivencia escolar

Se impulsa el intercambio de experiencias entre centros

Se han realizado programas de formación del profesorado en convivencia

El personal se implica en las acciones formativas para la mejora de la convivencia

Se participa en la celebración de actividades formativas conjuntas

Se realizan acciones formativas para las familias

Tabla 5. Escala: Formación de Docentes y Familiares

\section{Indicadores}

Se definen los principios de actuación pedagógica del aula de convivencia

Se establecen las condiciones de acceso

Se establece el procedimiento de derivación

Se planifica la organización del aula y sus recursos

Se definen "requisitos" del profesorado que atienda el aula

El centro asigna al resto de profesorado tareas de atención y control del aula

Se establece el horario de funcionamiento del aula

El departamento de orientación programa las acciones didácticas

Las personas responsables del aula realizan los seguimientos

El aula de convivencia tiene un tamaño adecuado

El mobiliario está dispuesto de forma que facilita el diálogo y la reflexión

Su ubicación la hace accesible y próxima

Existen materiales suficiente y de calidad para su uso didáctico

Tabla 6. Escala: Aula de convivencia 


\section{Indicadores}

El Plan de Convivencia ha contribuido a la colaboración entre el alumnado en el aula

,, a reducir las faltas injustificadas de puntualidad

„, , , las faltas injustificadas de asistencia

,, , , los daños en las instalaciones

, ,, los daños en las pertenencias

„, ,, a reducir los casos de maltrato

,, ,, el deterioro grave de las instalaciones

EI PC ha contribuido a mejorar las relaciones profesor-alumno

,, , las relaciones profesor-profesor

,, , las relaciones profesor-familia

, ,, las relaciones alumno-alumno

Tabla 7. Escala: Efectos

Para la validación teórica se toman dos medidas previas:

I) La revisión de literatura científica, experiencias, normativa y los planes experimentados sobre las acciones preventivas de estudio. Se utiliza los elementos que recoge tanto el Decreto 19/2007, de 23 de enero, por el que se adoptan medidas para la promoción de la Cultura de Paz y la Mejora de la Convivencia en los Centros Educativos sostenidos con fondos públicos. (BOJA n 25, del 2 febrero 2007) así como la Orden de 20 de junio de 20II, por la que se adoptan medidas para la promoción de la convivencia en los centros docentes sostenidos con fondos públicos y se regula el derecho de las familias a participar en el proceso educativo de sus hijos e hijas. (BOJA $n^{\circ} \mid 32$, del 7 de julio de 20 II).

2) Juicios de Expertos. Formado por seis miembros, profesionales de la orientación y personal docente e investigador de la Universidad de Huelva. Se procedió a la revisión de las escalas, por parte del grupo de expertos, valorando la pertinencia de los indicadores en cada una de ellas. El cuestionario se basa en una escala tipo Likert, donde se mide el grado de pertinencia, de 0 a 3 , siendo 0 nada pertinente, I algo pertinente, 2 pertinente, 3 muy pertinente. Tras su análisis fueron eliminados aquellos indicadores que menos puntuación habían obtenido. 
La fiabilidad mide la consistencia de los indicadores que forman el constructo, es decir, que los indicadores están midiendo lo mismo. Se calcula el Alpha de Cronbach y la fiabilidad compuesta. El Alpha de Cronbach permite analizar la fiabilidad de un conjunto de indicadores utilizados para medir un constructo, es habitual que en las investigaciones no aparezca implicado un solo constructo, sino varios. El Alpha de Cronbach para cada factor por separado no tiene en cuenta la influencia sobre la fiabilidad del resto de constructos. Por esta razón, Fornell y Larcker ( $198 \mathrm{I}$ ) proponen el cálculo de la fiabilidad compuesta (IFC) para cada factor que, interpretándose exactamente igual que el Alpha de Cronbach, sí que tiene en cuenta las interrelaciones. Los valores oscilan entre 0 (ausencia de homogeneidad) y I (máxima homogeneidad). Ambas propiedades alcanzan valores superiores al recomendable $(>0,70)$ en todos los constructos, estando comprendidos entre 0,86 y 0,97 .

\subsection{Análisis de correspondencias múltiples}

El ACM se ha realizado sobre una matriz de 70 variables con 350 modalidades de respuestas asociadas. El número de modalidades señaladas para las variables activas se refiere al obtenido después de los datos, consistente en eliminar, en todas las cuestiones activas, la falta de respuesta. Esta depuración es automática cuando las categorías presentan un escaso número de efectivos.

Todas las variables presentan cinco modalidades de respuestas, por lo que se mantiene cierto equilibrio en cuanto a la división de las variables en modalidades.

En general, los valores propios en este tipo de análisis ofrecen una idea pesimista de la variabilidad explicada, por lo que, como propone Benzécri (1979), pueden calcularse unas tasas de inercia más optimistas a partir de unos valores propios transformados. Para ello se utiliza la fórmula propuesta por este autor para transformar los valores propios de los cinco primeros factores

$$
a i_{\mathrm{i}}=\left(a i_{\mathrm{i}}{ }^{\prime}-\frac{1}{n^{\mathbf{0}} \text { modalidaes }-n^{\underline{0} \text { variables }}}\right)^{2}
$$

tras lo cual se halla el porcentaje de la varianza explicada por cada factor (varianza $=$ i / $\Sigma 5$ primeros valores propios transformados $\times 100$ ). 


\section{Resultados}

Se reducen los ítems de las 6 escalas en 5 factores. En el histograma se exponen los cinco primeros factores obtenidos en el análisis de correspondencias múltiples. Cada valor propio corresponde a la varianza explicada por cada factor que, como se aprecia en la siguiente tabla se mantiene prácticamente igual de un factor a otro. Se eligen los tres primeros factores para su análisis, pues explican casi el $82 \%$.

\begin{tabular}{|c|c|c|c|c|}
\hline Number & Value & Percentage & $\begin{array}{l}\text { Accumlated } \\
\text { Percentage }\end{array}$ & \\
\hline I & 0,1221 & 37.6 & 37.6 & 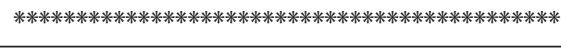 \\
\hline 2 & 0,0780 & 24 & 61.6 & 米米米米米米米米米米米米米米米 \\
\hline 3 & 0,0649 & 20 & 81.6 & 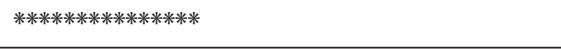 \\
\hline 4 & 0,0347 & 10.69 & 92.29 & **************** \\
\hline 5 & 0,0248 & 7.64 & 100 & 米米米米米米 \\
\hline
\end{tabular}

Tabla 8. Histograma de los cinco primeros factores

\section{I. Análisis factorial}

Factor I. Gestión del aula de convivencia y acciones de educación en el conflicto: En la sección negativa del eje factorial se localizan los valores bajos de las variables relativas a la inexistencia o escasa gestión del aula de convivencia y acciones de educación en el conflicto. Mientras en la sección positiva del eje factorial se sitúan los valores altos de las variables mencionadas.

Factor 2. Escasos efectos del plan de convivencia e inexistencia o escasa gestión del aula de convivencia y de acciones de educación en el conflicto: El factor 2 se articula entre variables relativas a los escasos efectos del plan de convivencia sobre la reducción de los diferentes tipos de problemas de convivencia así como la no existencia o desconocimiento del aula de convivencia y de acciones de educación en el conflicto, en la sección positiva del eje, y la escasa gestión de aula de convivencia y de acciones de educación en el conflicto en la sección positiva del eje.

Factor 3. Efectos del plan de convivencia y medidas de acciones preventivas: Este factor se integra, fundamentalmente, por modalidades de respuesta comprendidas, en la sección negativa, por valores bajos en variables relativas a los efectos del plan de 
convivencia sobre la reducción de los diferentes tipos de problemas de convivencia y con acciones para la promoción de la convivencia y, en la sección positiva, por aquellos valores más altos relativos a los efectos del plan de convivencia y medidas de acciones preventivas (elaboración de normas, educar en el conflicto, aula de convivencia).

\subsection{Resultados del análisis cluster}

El paso siguiente es identificar tipologías de centro para lo cual se presenta el gráfico donde figuran el factor I (eje horizontal) y el factor 3 (eje vertical). Se decide eliminar el factor 2.

Una vez establecidos los tres factores que sintetizan la información más relevante de la interrelación de las variables analizadas, se ha procedido a agrupar los sujetos en función de su afinidad respecto a las variables estudiadas. La clasificación en cuatro grupos o clases es la que se ha retenido finalmente por ser la que resultaba de mayor nivel explicativo.

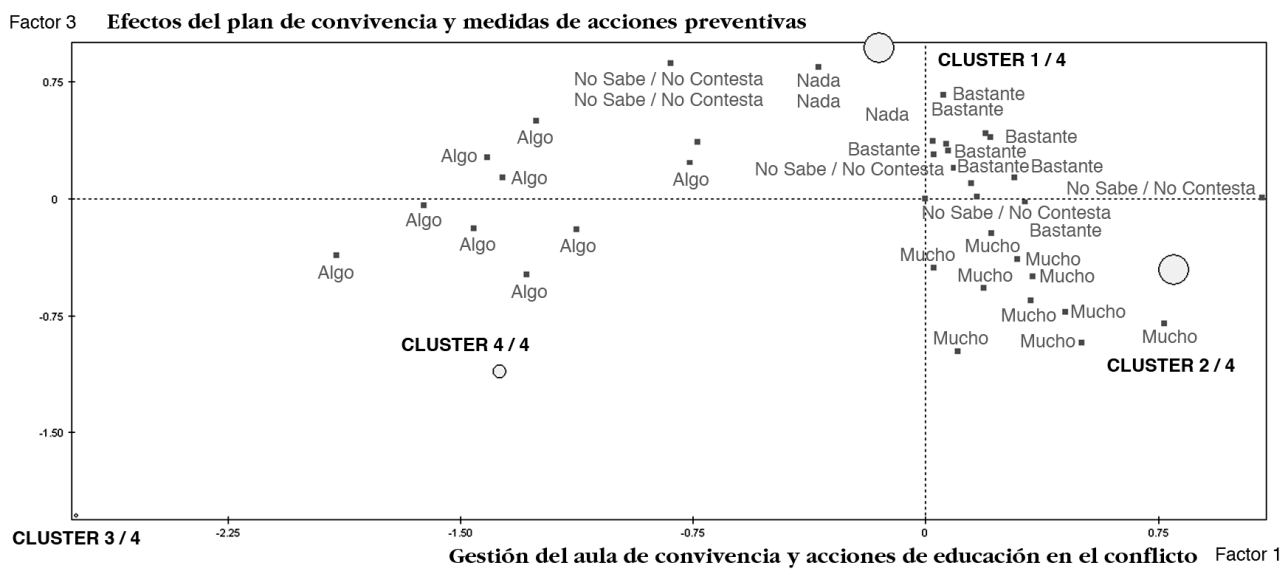

Figura I. Plano factorial de cluster

Tras el análisis factorial, se identifican cuatro clusters, entre los cuales se eligen tres, dado que uno de ellos se corresponde con un solo caso. Los cuatro clusters se describen a continuación (ver Figura I). 
- Cluster I. Desconocimiento de efectos o Efectos moderados del plan de convivencia y predominio de medidas de prevención. Se trata de una serie de casos $(4 I, 30 \%)$ en los que el profesorado manifiesta desconocer los efectos del plan de convivencia o efectos moderados del plan de convivencia sobre la reducción de los diferentes tipos de problemas de convivencia, con el predominio de ciertas medidas para la mejora de la convivencia en los centros educativos. Son centros en los que los indicadores relativos a la educación en el conflicto, elaboración de normas y aula de convivencia, alcanza valores altos.

- Cluster 2. Altos niveles en los efectos del plan de convivencia y predominio de medidas de prevención primaria. Se trata de casos (4I,30\%) en los que el profesorado manifiesta haber reducido en torno a bastante o mucho los problemas de convivencia. Existe cierto equilibrio entre el tipo de medidas adoptadas en niveles elevados, apareciendo un ligero predominio de la presencia de medidas de educación en el conflicto y aula de convivencia sobre formación de la comunidad escolar. Por otro lado se observa una ligera presencia de variables relacionadas con el liderazgo (recoger y discutir información de todos y con todos los miembros de la comunidad educativa; existencia de procedimientos para explicar y difundir el plan de convivencia).

- Cluster 4. Ausencia de reconocimiento del aula de convivencia con bajos niveles en los efectos del plan sobre la reducción de problemas y con escasez de medidas preventivas. En este grupo de centros (I5,22\%) se identifican modalidades de respuesta en las que no se reconoce la presencia del aula de convivencia. Asimismo, aparecen respuestas que indican bajos niveles en los efectos del plan de convivencia sobre la reducción de problemas de convivencia. También tiene presencia modalidades de respuestas que manifiestan desconocimiento o ausencia de medidas más preventivas.

Se ha elegido el factor I y el factor 3 para representar el plano factorial y proyectar los clusters (ver Figura I). El uso de ambos factores hace más visible la discrepancia entre conglomerados atendiendo a dos grupos de factores de interés en la investigación: los efectos del plan de convivencia sobre la reducción de problemas de convivencia respecto al liderazgo y la presencia de medidas preventivas de distinto carácter, sean relativas a la educación en el conflicto, relativas a la formación de la comunidad escolar o el aula de convivencia como medida de prevención terciaria. 


\section{Conclusiones}

A partir del análisis efectuado se han extraído las siguientes conclusiones:

En cuanto a la descripción de los factores que inciden en la mejora de la convivencia escolar se han reconocido tres factores: a) Factor I. Gestión del aula de convivencia y acciones de educación en el conflicto. En este sentido se coincide con otros estudios como los de Hendry (2010) y Maxwell (2007), donde ponen de manifiesto que la resolución de conflictos y los programas de mediación entre compañeros parecen ser eficaces para enseñar a los estudiantes habilidades de negociación y procedimientos de mediación, lo que llega a suponer una reducción del número de conflictos; b) Factor 2. Escasos efectos del plan de convivencia e inexistencia o escasa gestión del aula de convivencia y de acciones de educación en el conflicto y c) Factor 3. Efectos del plan de convivencia y medidas de acciones preventivas (Hendry, 2010 ; Maxwell, 2007; Jordan, 2009; Rodríguez, 2007; Serrano, Tormo y Granados, 20I I).

Respecto al segundo objetivo "identificar tipos de centros en función de los factores medidos por cada una de las escalas" se han obtenido tres tipologías: Cluster I. Desconocimiento de efectos o Efectos moderados del plan de convivencia, y predominio de medidas de prevención; Cluster 2. Altos niveles en los efectos del plan de convivencia y predominio de medidas de prevención primaria; Cluster 4. Ausencia de reconocimiento del aula de convivencia con bajos niveles en los efectos del plan sobre la reducción de problemas y con escasez de medidas preventivas.

En general, puede advertirse en el plano, atendiendo a los factores que lo configuran, que la reducción de problemas de convivencia en los centros educativos, aparecen asociados con altos valores en la gestión de medidas preventivas, sobre todo a nivel primario o secundario. Prueba de ello es la presencia del cluster 2 en la zona del primer cuadrante. Son también elevados los valores en las medidas preventivas del cluster 4, sin embargo, no lo son sus valores de satisfacción. El cluster 3 es el que presenta valores más bajos de satisfacción y, en cambio, moderados niveles de gestión del aula de convivencia, coincidiendo en este sentido con el cluster I. De todo ello se interpreta que el aula de convivencia es una medida preventiva no asociada a la satisfacción del personal y las familias con el plan de convivencia. Si bien, tiene presencia si está gestionada el aula de convivencia con un conjunto integral y equilibrado de acciones preventivas. 


\section{Referencias bibliográficas}

Andrés, S. y Barrios, A. (2009). De la violencia a la convivencia en la escuela: El camino que muestran los estudios más recientes. Revista Complutense de Educación, I, 205-227.

Ávila, J. A. (2013). El acoso escolar en Educación Primaria en la provincia de Huelva. Huelva: Universidad de Huelva.

Bodine, R. y Crawford, D. (1998). The Handbook of Conflict Resolution Education. A Guide to Building Quality Programs in Schools. The Jossey-Bass Education Series. Book, Whole. San Francisco: Jossey-Bass.

Burton, B. (20/2). Peer teaching as a strategy for conflict management and student re-engagement in schools. Australian Educational Researcher, 39, 45-58.

Cangas, A. J., Gázquez, J. J., Pérez, M. C., Padilla, D. y Miras, F. (2007). Evaluación de la violencia escolar y su afectación personal en una muestra de estudiantes europeos. Psicothema, 10, II4-119.

Casamayor, G. (2002). Cómo dar respuesta a los conflictos. Barcelona: Grao.

Conde, S. (2012). Estudio de la gestión de la convivencia escolar en centros de Educación Secundaria de Andalucía: una propuesta de evaluación basada en el Modelo EFQM. Huelva: Universidad de Huelva.

Decreto 19/2007, de 23 de enero, por el que se adoptan medidas para la promoción de la Cultura de Paz y la Mejora de la Convivencia en los Centros Educativos sostenidos con fondos públicos. (BOJA, N²5, del 2 Febrero 2007).

Díaz-Aguado, M. J., Martínez, R. y Martín, J. (20I0). Estudio Estatal sobre convivencia escolar en la Educación Secundaria Obligatoria. Observatorio Convivencia Escolar.

Eskildsen, J. K y Dahlgaard, J. J. (2000). A causal model for employee satisfaction. Total Quality Management, II, 8I-94.

Fernández-Enguita, M. (2007). Educar es cosa de todos: escuela, familia y comunidad. En J. Garreta (Ed.), La relación familia-escuela (pp. 13-32). Lleida: Edicions de la Universitat de Lleida.

Flynn, B., Schroeder, R. G y Sakakibara, S. (1994). A framework for quality management research and an associated measurement instrument. Journal of Operations Management, 4, 339-366. 
Fornell, C. y Larcker, D. F. (I98I). Evaluating structural equations models with unobservable variables and measurement error. Journal o Marketing Research, I8, 39-50.

García, L. y López, R. (20II). Convivir en la escuela. Una propuesta para su aprendizaje por competencias. Revista de Educación, 356, 53 I-555.

Hendry, J. (2010). Mediation in Schools: Tapping the potential. International Journal of School Disaffection, I, 26-32.

Johnson, D. y Johnson, R. (1996). Conflict resolution and peer mediation programs in elementary and secondary schools: A review of the research. Review of Educational Research, 66, 459-506.

Jordan, J. A. (2009). Cultura escolar, conflictividad y convivencia. Estudios sobre Educación, 17, 63-85.

Leithwood, K. (2009). ¿Cómo liderar nuestras escuelas? Santiago: Fundación Chile/ Fundación CAP.

Maxwell, J. (2007). Mediation in the schools: Self-regulation, self-esteem, and self-discipline. Conflict Resolution Quarterly, 2, I49-I55.

Orden de 20 de junio de $201 \mathrm{I}$, por la que se adoptan medidas para la promoción de la convivencia en los centros docentes sostenidos con fondos públicos y se regula el derecho de las familias a participar en el proceso educativo de sus hijos e hijas. (BOJA, $N^{\circ}$ I32, del 7 de julio de 20II).

Ortega, R., Romera, E. y Del Rey, R. (2005). Protagonismo de la atención a la convivencia en la prevención de la conflictividad y la violencia escolar. Información psicológica, 95, 4-I4.

Peligero, A. (2010). La prevención de la violencia desde el sistema educativo. Intervención Psicodidáctica en la desadaptación social: IPSE-ds, 3, 9-I7.

Pérez, V., Amador, L. V. y Vargas, M. (20II). Resolución de conflictos en las aulas: un análisis desde la investigación-acción. Revista Interuniversitaria de Pedagogía Social, 18, 99-II4.

Rodríguez, J. M. (2007). Medidas educativas que facilitan la resolución de conflictos. Docencia e Investigación. Revista de la Escuela universitaria de Magisterio de Toledo, 17, 265-282. 
Serrano, A., Tormo, M. P. y Granados, L. (20II). Las Aulas de convivencia, una medida de prevención a la violencia escolar. Criminología y Justicia, I, 4-8.

Sheerens, J. y Bosker, R. J. (1997). The foundations of educational effectiveness. Oxford: Pergamon.

Taffinder, P. (1995). The New Leaders: Achieving Corporate Transformation through Dynamic Leadership. London: Kogan Page.

Torrego, J.C. (20I0). La mejora de la convivencia en un instituto de educación secundaria de la Comunidad de Madrid. Profesorado. Revista de Currículum y formación del profesorado, I, 252-274. 